\title{
Particle Image Velocimetry Applications Using Fluorescent Dye-doped Particles
}

\author{
Brian J. Petrosky", Pietro Maisto ${ }^{\dagger}$, and K. Todd Lowe* \\ Virginia Polytechnic Institute and State University, Blacksburg, VA, 24060 \\ Matthieu A. André ${ }^{\S}$ and Philippe M. Bardet ${ }^{* *}$ \\ The George Washington University, Washington, DC, 20052 \\ and \\ Patsy I. Tiemsin ${ }^{\dagger \dagger}$, Christopher J. Wohl ${ }^{\dagger \dagger}$ and Paul M. Danehy $\$$ \\ NASA Langley Research Center, Hampton, VA,
}

\begin{abstract}
Polystyrene latex sphere particles are widely used to seed flows for velocimetry techniques such as Particle Image Velocimetry (PIV) and Laser Doppler Velocimetry (LDV). These particles may be doped with fluorescent dyes such that signals spectrally shifted from the incident laser wavelength may be detected via Laser Induced Fluorescence (LIF). An attractive application of the LIF signal is achieving velocimetry in the presence of strong interference from laser scatter, opening up new research possibilities very near solid surfaces or at liquid/gas interfaces. Additionally, LIF signals can be used to tag different fluid streams to study mixing. While fluorescence-based PIV has been performed by many researchers for particles dispersed in water flows, the current work is among the first in applying the technique to micron-scale particles dispersed in a gas. A key requirement for such an application is addressing potential health hazards from fluorescent dyes; successful doping of Kiton Red 620 (KR620) has enabled the use of this relatively safe dye for fluorescence PIV for the first time. In this paper, basic applications proving the concept of PIV using the LIF signal from KR620-doped particles are exhibited for a free jet and a twophase flow apparatus. Results indicate that while the fluorescence PIV techniques are roughly 2 orders of magnitude weaker than Mie scattering, they provide a viable method for obtaining data in flow regions previously inaccessible via standard PIV. These techniques have the potential to also complement Mie scattering signals, for example in multi-stream and/or multi-phase experiments.
\end{abstract}

\section{Nomenclature}

= Focal length

$=$ refractive index

$=$ magnification

$=$ bulk velocity

$=$ mean value

$=$ standard deviation

\footnotetext{
${ }^{*}$ Graduate Research Assistant, Dept. of Aerospace and Ocean Eng., 215 Randolph Hall, AIAA Student Member

${ }^{\dagger}$ Currently: Ph.D Candidate, Department of Mechanical Engineering, University of Maryland-College Park

${ }^{\ddagger}$ Assistant Professor, Dept. of Aerospace and Ocean Eng., 215 Randolph Hall.

${ }^{\S}$ Ph.D. Candidate, Mechanical and Aerospace Engineering, $80121^{\text {st }}$ St, NW, Washington DC 20052.

${ }^{* *}$ Assistant Professor, Mechanical and Aerospace Engineering, $80121^{\text {st }} \mathrm{St}$, NW, Washington DC 20052.

${ }^{\dagger}$ Research Scientist

tt Research Scientist, Associate Fellow AIAA.
} 


\section{Introduction}

$\mathrm{O}$ ne of the most widely used non-intrusive flow diagnostics tools that has been developed over the past few decades is Particle Image Velocimetry (PIV). PIV measurements are obtained by illuminating a particle-seeded flow with a laser sheet and recording a series of images of the particles in the flow. By tracking the movements of these particles, velocity fields of the flow can be mapped. This technique allows for accurate velocity measurements at any point in the flow illuminated by the laser, and has had numerous applications in studying both laminar and turbulent flows [1]. A common type of seed particle that has been used for PIV measurements is Polystyrene Latex (PSL) microspheres [2]. They are advantageous because of their small size and low density, which allows them to faithfully follow the actual flow around them. Also, their high index of refraction greatly enhances their visibility in PIV imaging.

Recent work at NASA Langley Research Center has refined the manufacturing techniques of these spherical microparticles to create a highly uniform size distribution on the order of $1 \mu \mathrm{m}$ [3]. Furthermore, these particles can be doped with a fluorescent dye to exhibit Laser Induced Fluorescence (LIF). When fluorescent dye-doped particles are excited by incident laser light, they both scatter the light at the same wavelength as the incident laser light (Mie scattering) as well as emit light that is red-shifted relative to the incident lightthe Stokes shift. For example, see the emission spectra in Fig. 1 for the Kiton Red 620 (KR620) dye used for current work. The peak fluorescence wavelengths of KR620 occur between 580 and 600 $\mathrm{nm}$. A long pass filter with a cut-on wavelength between $532 \mathrm{~nm}$ and these peak fluorescence wavelengths can be used to block all Mie scattering and only transmit fluorescent light. One may utilize the LIF signal for a variety of applications, including simultaneous temperature and velocity measurements

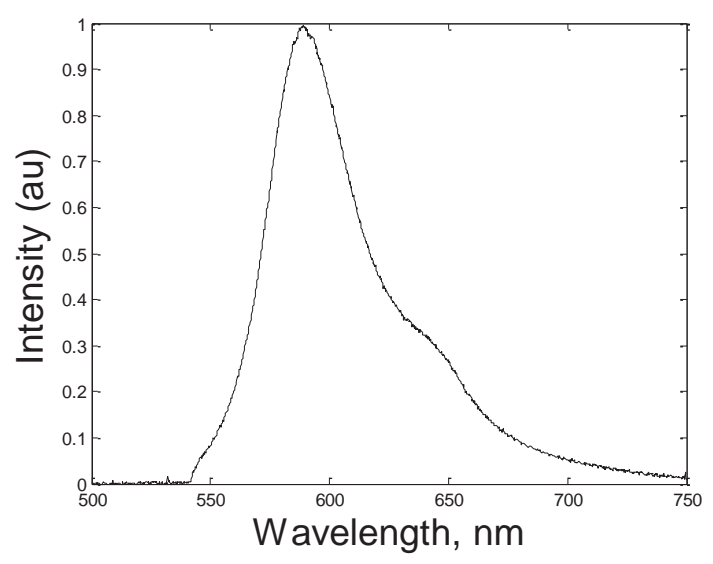

Figure 1. Measured emission spectrum of Kiton Red 620 doped PSLs collected from excitation with a continuous-wave laser at $532 \mathrm{~nm}$. A spectral filter blocked the laser light.

$[4,5]$. In the majority of applications, PIV data are taken using the Mie scattered light from the seed particles, while LIF is utilized by a second camera to obtain flow temperatures $[4,5]$. Using fluorescent light for PIV, rather than Mie scattering, has led to a variety of applications in water flows [6, 7]. However, little research has been performed to demonstrate the reliability or applicability of the concept in air, which requires smaller particles and safe dyes due to inhalation concerns. A past effort in an air flow was successful, but utilized potentially dangerous seed particles that required protective measures for operation [8].

The use of fluorescent light for PIV has the potential to be especially useful in liquid-gas flows, where laser light is reflected and refracted at the interface. This creates glare on the images when stray laser light reaches the sensor [9]. Glare is detrimental because it decreases the signal to noise (SNR), and saturates the sensor pixels, which can lead to signal leakage onto neighboring pixels and even result in sensor damage. In general, data in the presence of a glare are of low quality, if usable at all. Similar challenges also arise when the laser light is scattered by a solid object in the field of view, such as a solid boundary.

Much of the past work with LIF or dye-doped PSL's has involved the use of phosphor particles containing rare earths or other chemicals such as toluene or Rhodamine $[10,11,12,13]$. These chemicals can be dangerous and are unsafe to users in large flow facilities. The present work brings together multidisciplinary efforts from teams of researchers at NASA Langley Research Center, George Washington University and Virginia Tech with a focus on finding and applying safe fluorescent dyes to dope seed particles that can be used for LIF and flow diagnostics, such as PIV or Laser Doppler Velocimetry (LDV). Past work has centered around Dichlorofluorescein (DCF) and KR620 dye-doped PSL's as safe alternatives for LIF, with Kiton Red exhibiting promising results [2, 14, 15]. The KR620 doped PSL particles used in the present work had 0.5 micron diameter and were developed using an improved proprietary version compared to that initially described in [15]. 


\section{Instrumentation and Test Procedure}

\section{A. Free Jet Experiments in Air}

The set of experiments took place at Virginia Tech's Vortical Flow and Diagnostics Lab (VTFD). They involved PIV data taken using a LaVision Imager Pro X 4M CCD camera with a 2048 x 2048 pixel resolution and processed using LaVision's DaVis software, Version 7.2 [16]. Fig. 2 shows the setup used for all VTFD testing. The single camera (1) was positioned directly over the nozzle exit (2) and allowed for 2D velocimetry. A Sigma $105 \mathrm{~mm} \mathrm{f} / 2.8$ EX DG macro lens (3) was used with the camera to obtain a close-up image of the flow. For fluorescence imaging, an Omega Optical $560 \mathrm{~nm}$ long pass filter (model 560HLP ) was attached to the lens, blocking out all Mie scattered light from the particles and only allowing particle-emitted fluorescent light to be captured by the camera. A $532 \mathrm{~nm}$ double-pulsed Nd:YAG laser (4) was used at approximately $200 \mathrm{~mJ} /$ pulse to illuminate the flow and was controlled simultaneously with the camera by the DaVis software, recording at about $10 \mathrm{~Hz}$. Finally, an $\mathrm{f}=-25 \mathrm{~mm}$ cylindrical lens (5) was used to form a thin laser sheet at the nozzle exit as indicated in Fig. 2.

The KR620-doped PSL particles were seeded using an Air-o-Swiss 7146 Ultrasonic Humidifier with a variable output setting to control how much seed was introduced into the flow. This vaporizer was placed in a mixing chamber, where it mixed with the air and flowed through a tube and finally a custom nozzle with an area ratio of 14.4:1 and exit diameter of $\mathrm{d}$ $=14.15 \mathrm{~mm}$. The laser sheet extended approximately $3.15 \mathrm{~d}$ from the nozzle exit. Finally, flow velocity was controlled using a Forney 75546 Air Line Mini-Regulator. Test velocities were measured using a Pitotstatic probe and Dwyer Series 475 Mark III manometer, which were then compared to the PIV processed data. Before each test, 50 $\mathrm{mL}$ of the KR620 particle solution were

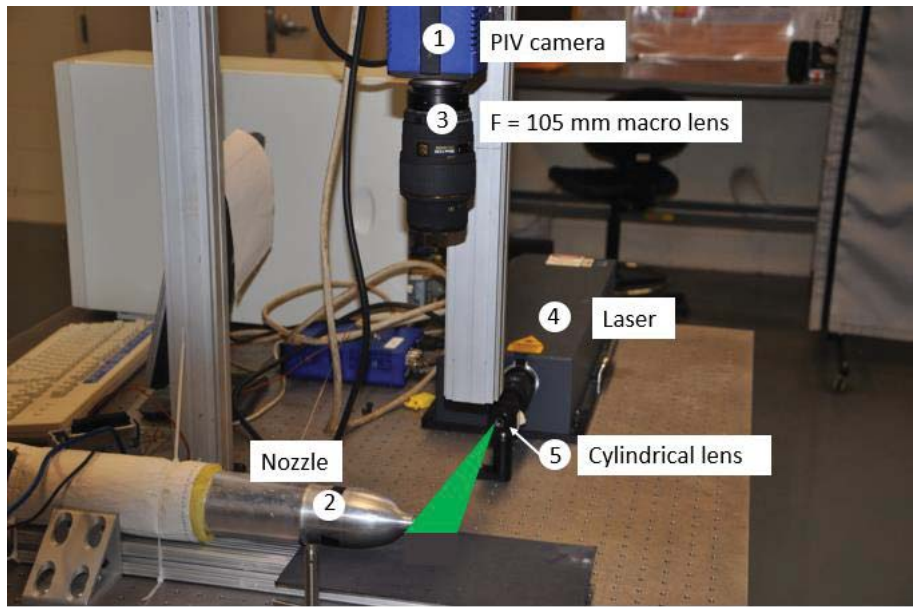

Figure 2. Experimental PIV test setup at Virginia Tech. mixed with $50 \mathrm{~mL}$ of distilled water in an L\&R Quantrex $90 \mathrm{H}$ ultrasonic disruptor to prevent particle agglomeration. The mixture was then removed from the disruptor and placed immediately into the vaporizer. Tests were run at low speeds, on the order of $3.5 \mathrm{~m} / \mathrm{s}$, a speed that enabled optimal seeding densities for the vaporizer. During each test, 15 image sets were captured with the 560 -nm filter on the lens with a lens f-stop of $\mathrm{f} / 2.8$. Immediately afterwards, the filter was removed and 15 additional Mie scattering images were taken with a lens f-stop of $\mathrm{f} / 22$. All tests were run at room temperature. Velocity vectors were obtained via cross-correlation of the two images in each set, using a multi-pass technique in the DaVis software with $64 \times 64$ pixel initial and $32 \times 32$ pixel final interrogation windows and $50 \%$ overlap.

\section{B. Two Phase Experiments}

The two-phase experiments were conducted at George Washington University in a test facility that consisted of a rectangular water jet flowing from a contoured nozzle onto a transparent channel. Special care was taken during the design of this facility to minimize the laminar boundary layer thickness exiting the nozzle and to increase the uniformity of the bulk of the flow. The top boundary layer, which becomes a shear-layer upon leaving the nozzle, has a small thickness $(<1 \mathrm{~mm})$ compared to the jet depth $(20.3 \mathrm{~mm})$ and width $(146 \mathrm{~mm})$. Therefore, the channel bottom and side walls, which allow optical access, have a negligible influence on the surface near the mid-width of the channel. This simplified flow is initially equivalent to a semi-infinite 2D flow. The reader can refer to [17] for a detailed description of the apparatus. 
The flow is probed using PIV. The use of highspeed cameras (Phantom V-series) and a high repetition rate laser (Dual-cavity, frequency-doubled Nd:YLF, $527 \mathrm{~nm}$ at up for double pulse operation at $10 \mathrm{kHz}$ ) allow resolving the flow with a temporal resolution of $100 \mu$ s. High magnification optics (up to $\mathrm{M}=4$ ) provide the required spatial resolution (less than $100 \mu \mathrm{m}$ between velocity vectors). The field of view is around one centimeter wide in the streamwise direction. The facility offers the possibility to measure the velocity field in the liquid phase and/or in the gas phase, using two cameras. The laser light is delivered from the side which is imaged to avoid deformation of the light sheet by the interface, seen in Fig. 3.

A first study made use of non-fluorescent $2 \mu \mathrm{m}$ diameter PSL particles [3]. A second study made use of $0.5 \mu \mathrm{m}$-diameter PSL particles doped with KR620, manufactured in a manner similar as described in [14]. The tracers are in a water solution placed in an atomizer (TSI six-jet 9306) and introduced in the air above the outlet of the nozzle. The injection velocity is kept as low as possible to minimize disturbances on the gas flow, while providing sufficient seeding density. The particles follow the air entrained by the liquid jet surface. Fig. 3 shows the test section configuration. The profile of the surface must also be recorded in order to process the PIV data. The large amplitude surface deformation does not allow a precise measurement of the interface location from the PIV particle images alone due to reflections. Therefore, rhodamine 6G (R6G) - a fluorescent dye - is homogenously dissolved in the liquid. A camera viewing from above the surface detects the interface as a sharp change in light intensity. This top camera also records the PIV signal in the gas phase. Liquid phase measurement methodology and results using reflective particles are presented in [18]. Simultaneous gas/liquid measurements are presented in [19].

\section{Results and Discussion}

\section{A. Free Jet Results in Air}

Multiple sets of tests were run in the VTFD Lab on fluorescence PIV signal quality from the free jet. Pictured in Fig. 4 are raw single-shot images captured by the PIV camera showing the flow at the exit of the nozzle exhaust. The top image was taken without the laser-blocking filter and shows the Mie scattered light. The bottom image was taken using the $560-\mathrm{nm}$ long pass filter and therefore only contains fluorescent light emitted by the KR620 particles. The particles are about 2 orders of magnitude brighter in the unfiltered Mie scattering images compared to the LIF images, requiring different apertures to view the flow while holding laser illumination constant. Nonetheless, the overall flow instability visualization is still clearly visible with the fluorescent light and results in a well-seeded image of the core jet flow. The flow is from right to left in these images and was measured at the nozzle exit via the Pitot-static probe and manometer to be $3.5 \mathrm{~m} / \mathrm{s}$. The signal intensity in the right-hand side of the image in Fig. $4 \mathrm{~b}$ is caused by a reduced sensitivity of the right half of the sensor which was not working properly and which. This was an artifact of the experiment that is exacerbated by the lower signals in the fluorescent PIV images.

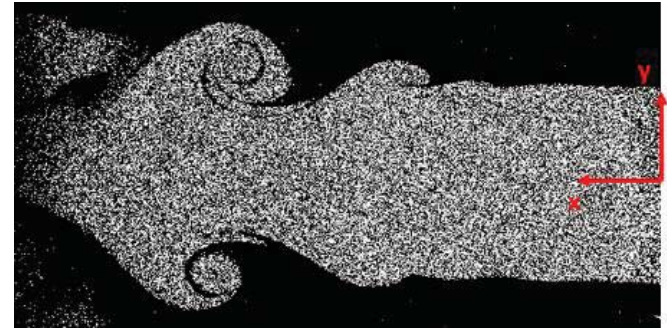

(a)

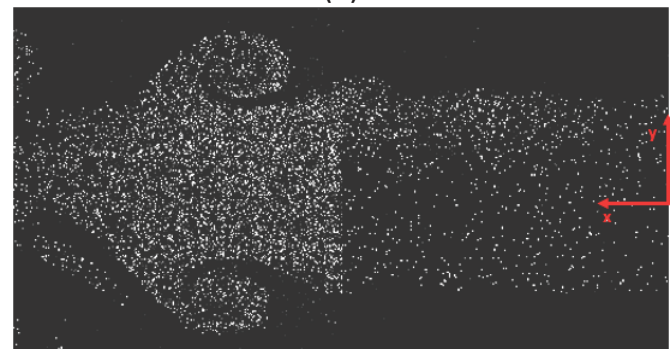

(b)

Figure 4. Camera images of Mie scattered light (a) and KR620 fluorescent light (b). Images were taken at a nozzle exit velocity of $3.5 \mathrm{~m} / \mathrm{s}$. The images were not taken simultaneously. 
The image sets were processed using the $\mathrm{DaVis}$ software to obtain particle velocity vectors. The velocity maps obtained with Mie scattered data are plotted in Fig. 5. The original image from Fig. 5a, the processed U- and Vvelocities (along the $\mathrm{x}$ - and $\mathrm{y}$ - axes as labeled in Fig. 4), and the average U- velocity from a set of 15 such images are contained in this figure. The same four plots obtained using the KR620-doped particles are shown in Fig. 6.

These initial test results lead to a few conclusions. First, the Mie-processed U-velocity average from Fig. 5d matches the measured manometer flow velocity of $3.5 \mathrm{~m} / \mathrm{s}$ within instrumentation uncertainties, which validates the accuracy of the PIV data. Second, both Mie and fluorescent processed images can successfully measure detailed flow features seen in the individual images. For example, the radial velocities expected from the ring-vortex instabilities seen in Figs. 5a and 6a can be clearly seen in both Figs. 5c and 6c. Third, and most importantly, the Uvelocity average from the KR620 fluorescent images in Fig. 6d is within 3\% of the U-velocity average of the Mie scattered images at the nozzle exit. Because the sets of images were taken back to back, rather than simultaneously, it is unknown whether the slight difference represents an instrumentation bias in PIV data from fluorescent images or reflects an actual velocity change in nozzle exit velocity from one set of flow images to the next. However, this difference is relatively small, and it is strongly believed that the fluorescent and Mie PIV data are consistent. Thus, the Virginia Tech results serve to validate the use of the dye-doped particles to measure the same velocity field as the Mie scattering method, at least to within 3\% for this flow.

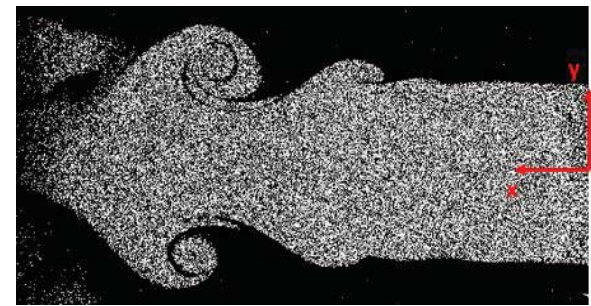

(a)

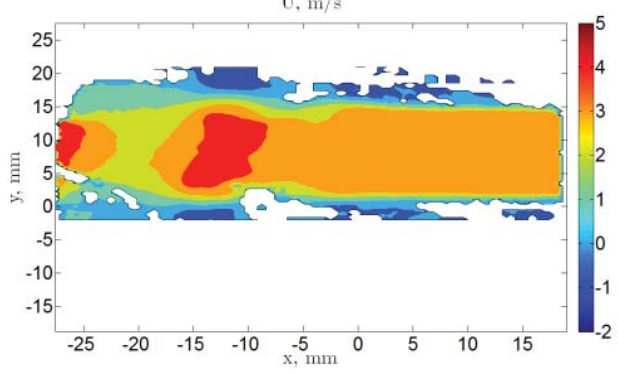

(b)

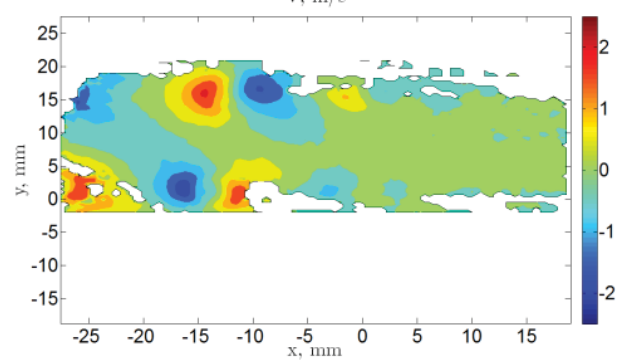

(c)

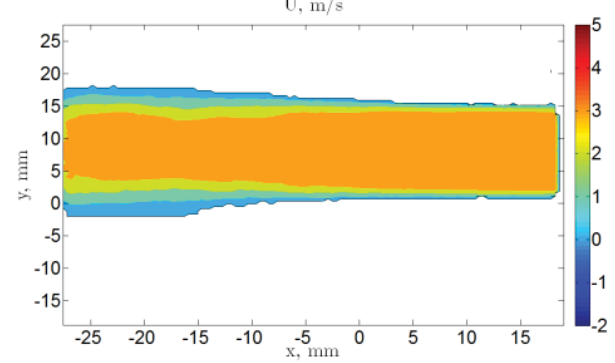

(d)

Figure 5. Original camera image of Mie scattered light (a), single image processed PIV data of $U$ - and V- velocities (b and $c$ ), and 15 image average $U$ - velocity (d).

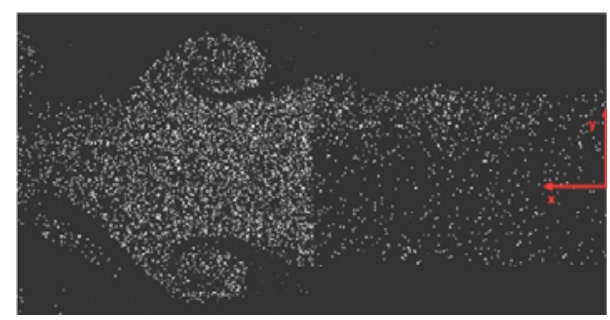

(a)

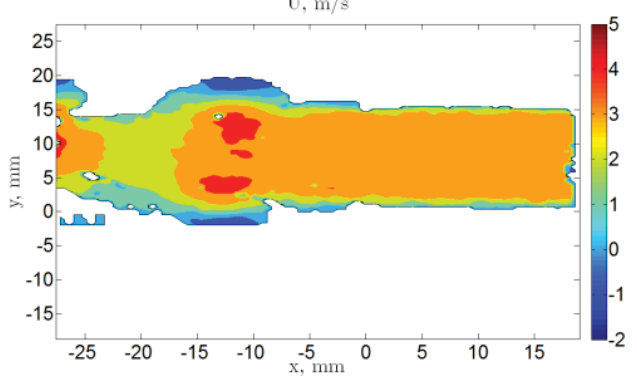

(b)

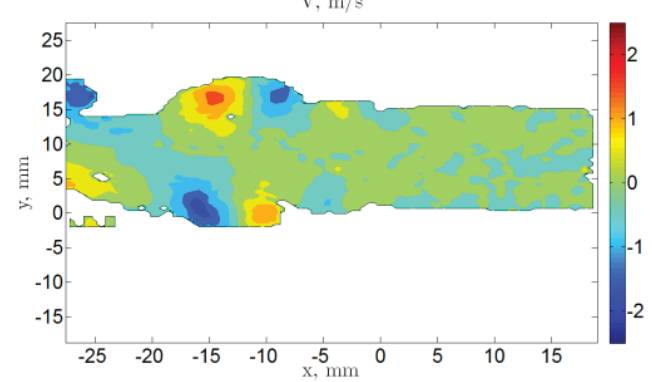

(c)

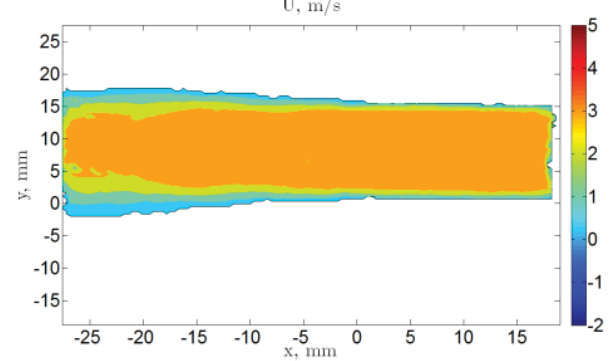

(d)

iu Figure 6. Original camera image of KR620 fluorescence (a), single image processed PIV data of $U$ - and $V$ - velocities (b and c), and 15 image average $U$ - velocity (d). 


\section{B. Two Phase Results}

When non-fluorescent tracers are used (Mie scattered light only), no spectral filter is mounted on the imaging camera. Therefore, the sensor will collect light from each of (1) the laser, (2) liquid phase particles, (3) R6G dye dissolved in the liquid and (4) gas phase particles. Of these four signals, only the last two constitute the actual signals of interest. The first two are noise. Secondary reflections and diffuse laser light will also produce secondary signals that are undesirable. Fig. 7 shows an example of a raw image using undoped particles. Several image defects are visible such as the reflection of a laser beam at the surface. The glare there locally saturates the sensor, which prevents resolving the surface profile and the flow field in this region. Other defects are visible in the background of the image, caused by secondary reflections. This example features a surface with low deformations; frames without reflection can still be found and manually selected for processing. Tracers in the liquid phase are also visible. Such tracers in the bulk of the liquid are not problematic, as this part of the image is not used. However, when they are near the surface, their signal introduces noise to the R6G signal.

The performance of PIV also depends on the SNR of the particles [20]. The SNR is defined in this application as

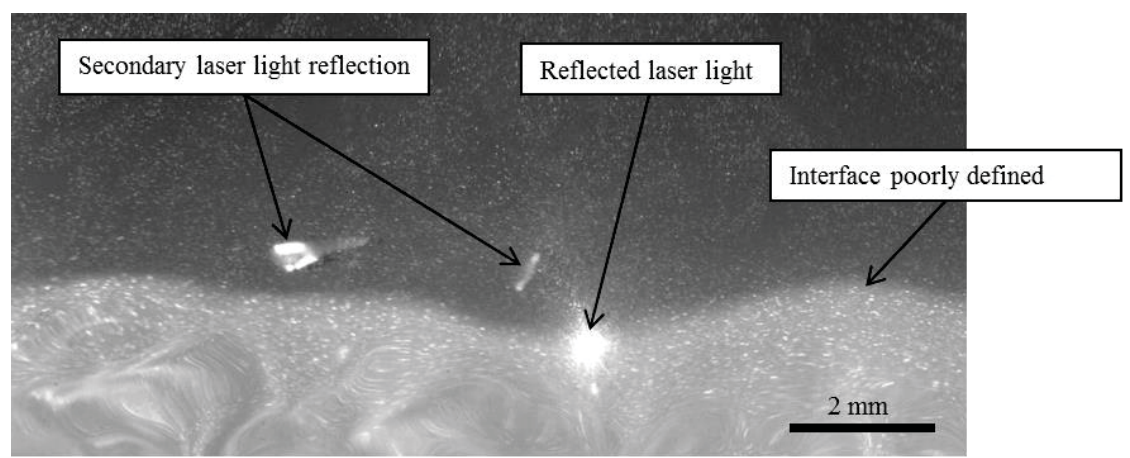

Figure 7: Raw image using reflective particles. $M=1.35,2.6 \mathrm{~mJ} /$ pulse, $\mathrm{f} / 11 . \mathrm{U}=2.46 \mathrm{~m} / \mathrm{s}$.

$$
S N R=\frac{\mu_{\text {signal }}}{\sigma_{\text {background }}}
$$

where $\mu$ and $\sigma$ are the mean and standard deviation, respectively. The SNR can be express in $\mathrm{dB}\left(20 \log _{10}(S N R)\right)$ or in bits $\left(\log _{2}(S N R)\right)$. The quantization error is negligible for SNR $>16 \mathrm{~dB}$ (or 4 bits) [20]. In Fig. 7, the particles have an SNR of $30 \mathrm{~dB}$ or 5 bits, which is sufficient for PIV. Note that M is magnification and $\mathrm{U}$ is the water velocity.

In summary, the reflective particles offer a good SNR, and a good response in regions of low acceleration. However, reliable results cannot be obtained near the surface. High accelerations induce a non-negligible slip velocity. More importantly, imaging at the same wavelength as the illumination source results in intense glare. This glare can prevent PIV processing, impede the measurement of the surface profile, and can also damage the camera.

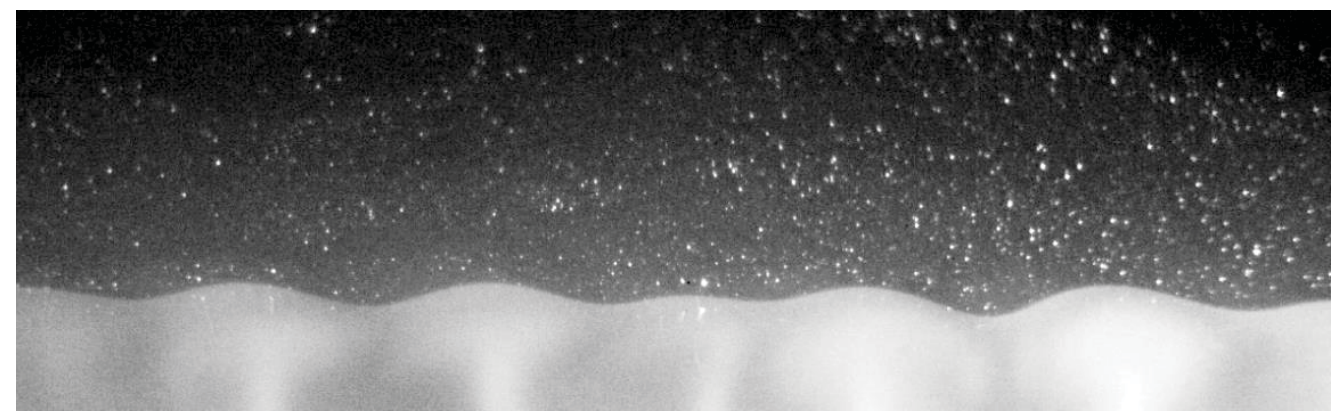

Figure 8: Raw image using fluorescent particles. $M=1.8,4 \mathrm{~mJ} /$ pulse, $\mathrm{f} / 2.8$. $\mathrm{U}=2.72 \mathrm{~m} / \mathrm{s}$. Contrast adjusted for clarity.

Next, the tests were performed again using KR620-doped fluorescent particles. The laser power had to be increased to $4 \mathrm{~mJ} / \mathrm{pulse}$, which is the maximum for the present laser at $10 \mathrm{kHz}$, and the lens $\mathrm{f}$-number had to be changed to 2.8 in order to get an SNR of 4 bits. Fig. 8 shows a raw PIV image using fluorescent particles and long pass filters. Laser reflection and particle images in the liquid phase have been totally filtered out. Some near-surface particles in the gas phase are reflected in the liquid, but their number is limited and do not affect the interface detection scheme. By using fluorescent light from the particles, the gas-water interface is clear and glare has been eliminated. 
The large lens aperture results in a narrow depth of focus, especially at this high magnification. The depth of focus is around $0.04 \mathrm{~mm}$. This is smaller than the laser sheet thickness $(0.2 \mathrm{~mm})$ so some particles are out of focus. The focus is optimized near the interface, but because the camera is angled $\left(9.3^{\circ}\right)$, particles in the upper part of the field of view are not in focus (this can be improved with a Scheimpflug lens mount). Fig. 8 shows a sharp interface and small particle images (diffraction limited) near the interface, and larger particles in the bulk. This is deemed acceptable since the focus of this work is on the near-surface velocity field. Out of focus particles still provide an acceptable signal for measuring the flow field.

Fig. 9 presents a vector field obtained using fluorescent particles. Details of the processing can be found in [19]. Such data give insight into interfacial shear in the gas phase.

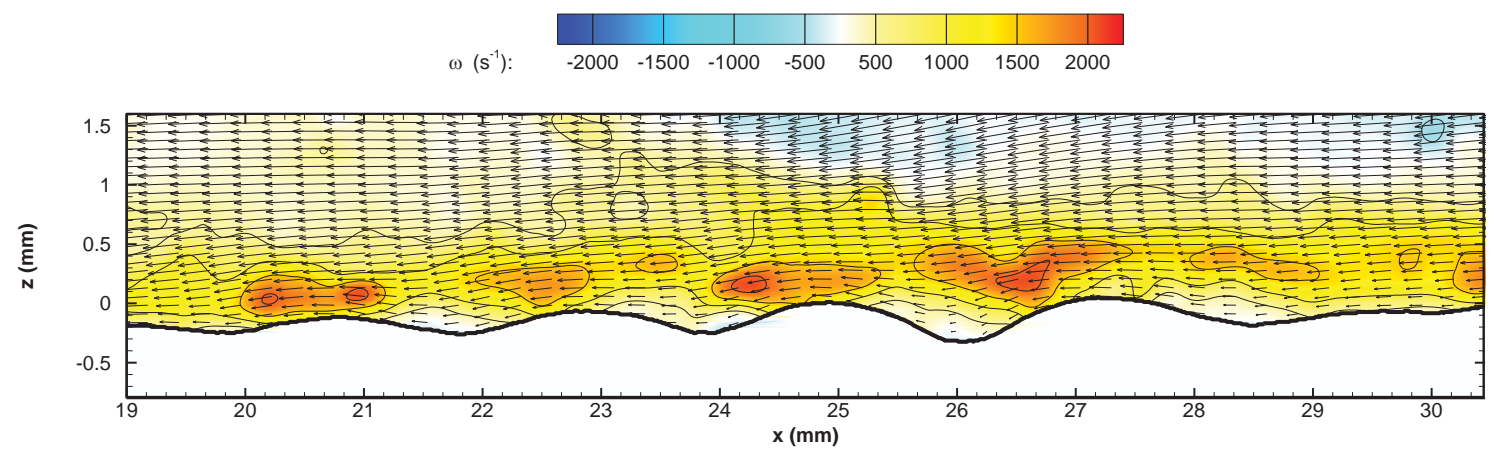

Figure 9: Process PIV frame using fluorescent particles. Velocity field and vorticity contour plot for $U=2.72 \mathrm{~m} / \mathrm{s}$.

\section{Conclusion}

These results demonstrate the applicability of fluorescent PIV using KR620 doped PSL particles. Tests at Virginia Tech's VTFD lab showed consistent results between PIV data taken in an air flow using the traditional Mie scattered light and PIV data taken using only the fluorescent light of the KR620-doped particles. Work at George Washington University involving flow at an air/water interface provided an example application where using fluorescent light for PIV is necessary to obtain a velocity field in areas of the flow near a potentially scattering surface. The KR620 doped PSL particles used for these experiments are small enough to accurately follow the flow of air around it as well as relatively safe for use in a large, open air flow facilities.

\section{Acknowledgments}

The authors acknowledge the support of the NASA ARMD Seedling Fund and NIA Cooperative Agreement NNL09AA00A.

\section{References}

1. Adrian, R. J., "Twenty Years of Particle Image Velocimetry,” Experiments in Fluids, Vol. 39, 2005, pp. 159-169.

2. Maisto, P. M. F., "Experimental Analysis and Prospective Flow Diagnostic Applications for Fluorescence Dye-Doped Micro-Particles,” M.S. Thesis, Aerospace and Ocean Engingeering Department, Virginia Tech, Blacksburg, VA, 2014.

3. Tiemsin, P., and Wohl, C. "Refined Synthesis and Characterization of Controlled Diameter, Narrow Size Distribution Microparticles for Aerospace Research Applications," NASA Technical Memorandum, 2012, pp. TM-2012-217591.

4. Peterson, B., Baum, E., Böhm, B. Sick, V, and Dreizler, A., "Evaluation of Toluene LIF Thermometry Detection Strategies Applied in an Internal Combustion Engine," Applied Physics B, April 2014.

5. Abram, C., Fond, B., Heyes, A. L., and Beyrau, F., "High-speed Planar Thermometry and Velocimetry Using Thermographic Phosphor Particles," Applied Physics B, April 2014, Vol. 111, 2013, pp. 155-160.

6. Fu, T. C., Bing, R., and Katz, J., "Automatic Particle-Image Velocimetry Utilizing Laser-Induced Fluorescent Particles," The Proceedings: Fifth International Conference on Numerical Ship Hydrodynamics. Washington D.C.: The National Academies Press, 1990, pp. 493-498.

7. Poussou, S., and Plesniak, M. W., "Near-Field Flow Measurements of a Cavitating Jet Emanating From a CrownShaped Nozzle", Journal of Fluids Engineering, Vol. 129, No. 5, 2006, pp. 605-612.

8. Chennaoui, M., Angarita-James, D., Ormsby, P. M., Angarita-James, N., McGhee, E., Towers, C. E., Jones, A.C., and Towers, D. P., "Optimization and Evaluation of Fluorescent Tracers for Flare Removal in Gas-Phase Particle Image Velocimetry," Measurement Science and Technology, Vol. 19, No. 11, 115403, 2008. 
9. Bardet, P., Peterson, P., and Savas, O., Split-screen single-camera stereoscopic PIV application to a turbulent confined swirling layer with free surface. 2010, Exp. Fluids, Vol. 49, pp. 513-524.

10. Omrane, A., Peteron, P., Aldén, M., and Linne, M. A., "Simultaneous 2D Flow Velocity and Gas Temperature Measurements Using Thermographic Phosphors." Applied Physics B., Vol. 92, 2008, pp. 99-102

11. Brübach, J., Patt, A., and Dreizler, A., "Spray Thermometry Using Thermographic Phosphors," Applied Physics B, Vol. 83, 2006, pp. 499-502.

12. Omrane, A., Särner, G., and Aldén, M., “2D-Temperature Imaging of Single Droplets and Sprays Using Thermographic Phosphors," Applied Physics B, Vol. 79, 2004, pp. 431-434.

13. Petracci, A., Delfos, R., and Westerweel, J., "Combined PIV/LIF Measurements in a Rayleight-Bénard Convection Cell”, $13^{\text {th }}$ International Symposium on Applications of Laser Techniques to Fluid Mechanics, Lisbon, Portugal, June 2006, Paper \#1221.

14. Maisto, P., Lowe, K. T., Byun, G., Simpson, R., Verkamp, M., Danehy, P., and Tiemsin, P., "Characterization of Fluorescent Polystyrene Microspheres for Advanced Flow Diagnostics," AIAA AMT Conference, San Diego, CA., 2013.

15. Danehy, P., Tiemsin, P., Wohl, C., Verkamp, M., Lowe, K. T., Maisto, P., Byun, G., and Simpson, R., "FluorescenceDoped Particles for Simultaneous Temperature and Velocity Imaging," NASA Technical Memorandum, Sept. 2012, pp. TM-2012-217768.

16. Davis Software Package, Ver. 7.2., LaVision, Goettingen, Germany, 2006.

17. André, M. A., and Bardet, P., Experimental Investigation of Boundary Layer Instabilities on the Free Surface of NonTurbulent Jet. Proceedings of the ASME FEDSM. 2012.

18. André, M. A., and Bardet, P., "Velocity field and surface profile resolution below steep and short surface waves," Experiments in Fluids. 2014.

19. André, M. A., and Bardet, P., "Interfacial shear measurement using high resolution multiphase time resolved PIV," The $17^{\text {th }}$ International Symposia on Applications of Laser Techniques to Fluid Mechanics, Lisbon, Portugal, July 2014.

20. Adrian, R and Westerweel, J. Particle Image Velocimetry. s.1. : Cambridge University Press, 2010. 